\title{
Solar energy at high temperatures; researches at the Weizmann Institute of Science, Israel; 25 years of success
}

\author{
Akiba Segal* \\ Center of Energy Research, Faculty of Chemistry, Weizmann Institute of Science, Rehovot, Israel
}

\begin{abstract}
The paper describes the features of the solar complex at the Weizmann Institute of Science and the projects successfully realized during its 25 years of operation.
\end{abstract}

\section{Introduction}

The sun is equivalent to a $5800-\mathrm{K}$ thermal reservoir. If we were able to concentrate sunlight on the earth, close to the thermodynamic limit, and to use its power almost without loss, we would obtain the most powerful thermal engine with fantastic efficiency of more than $90 \%$. Unfortunately, this seems to be impossible and we must be content with much less. It follows, for thermodynamic reasons, that the efficiency increases considerably using systems working at high temperature. But the high temperature is not the unique parameter that influences the efficiency of a solar heat system. In Figure 1 is shown the efficiency of a solar heat thermal collector, receiving solar energy at various temperatures and at various concentrations. It can be seen that maximum efficiency is obtained at a temperature depending on the concentration, and increases when the concentration increases.

A great deal of research is put into harvesting and storage of solar energy for power generation, using thermal power with the best efficiency. Concentrated solar power has been extensively investigated in the last four decades and it is based on a general scheme: using mirrors, sunlight can be redirected, focused and collected as heat, which is then used to activate a turbine or a heat engine to generate electricity. There are two principal categories of solar concentrated power plants utilized for this purpose: the parabolic trough plant and solar tower power plant. It is also worth mentioning two other technologies less used on large scale: linear Fresnel concentrators and parabolic dishes [1]. In parallel, the concentrated solar power can be used with remarkable success for various chemical reactions requiring high temperatures.

An experimental solar tower with heliostats field was constructed at the Weizmann Institute of Science (WIS) in 1988 and has served for a quarter of a century as the main center for research of concentrated solar energy in Israel. The WIS tower (height: $56 \mathrm{~m}$ ) comprises a North field of

* e-mail: akiba.segal@weizmann.ac.il
64 heliostats, $54 \mathrm{~m}^{2}$ reflective surfaces (the heliostats field layout is shown in Fig. 2), which track the sun individually and reflect the light onto a selected target located on each of five experimental levels of a solar tower (Fig. 3). Light can be reflected toward any or all of these testing stations, allowing a number of experiments to be carried out simultaneously.

This solar tower facility, located on the campus of a research and academic institute and dedicated solely to scientific work, is unique in the world. This paper describes the most important projects that were successfully realized during its 25 years of operation [2]. Our goal was to explore solar-driven thermal and chemical processes, enabling power production, fuel alternatives, long-term storage and convenient transportation options. Work at WIS is diverse and evolves based on the scientists' vision and imagination. The projects, during that period, were dedicated to researching experimental systems for developing cost-effective ways for environmentally clean, solar-driven electricity production, methane reforming, methane cracking, hydrogen production, solar energy storage, pressurized air heating for solarized Brayton cycle and different thermochemical reactions, solar energy storage, redox cycles for water splitting and hydrogen production at atmospheric pressure and under vacuum conditions and solar reduction of metal oxides, for example, the production of zinc from zinc oxide, for developing a clean process to provide zinc for fuel cells and for the production of hydrogen.

\section{First projects and first success}

At the beginning of 1989, the first pilot was run for producing saturated steam at maximum nominal rate of 2.3 ton per hour at 15 bars and about $500 \mathrm{~K}$ [3]. For the first time in Israel, a thermal system was put into operation, powered by solar energy, using solar tower technology. This project successfully demonstrated the concept of physical separation between the boiling and superheating sections for safe and efficient operation of a solar steam generator. Our team gained experience to effectively operate the 


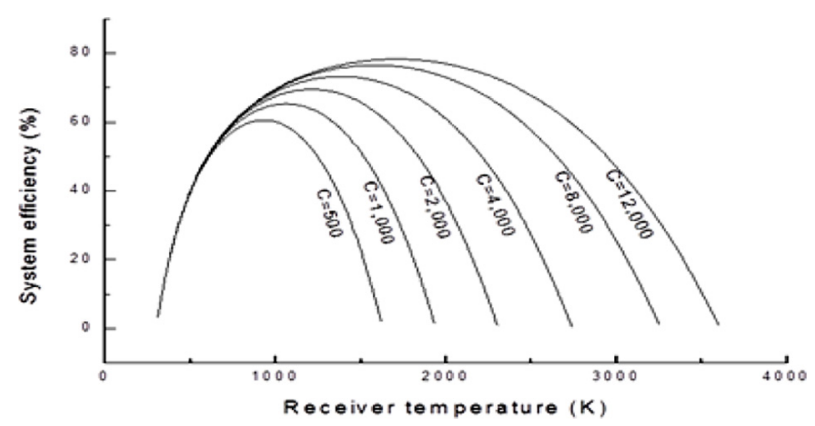

Fig. 1. System efficiency at various concentrations.

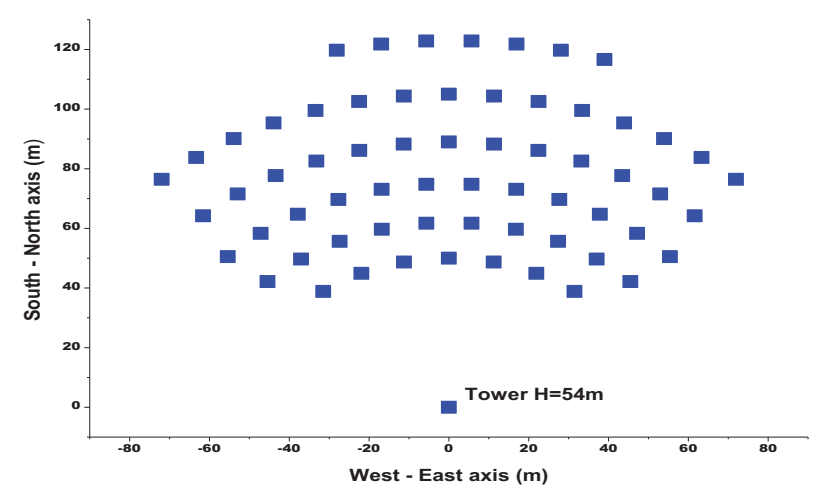

Fig. 2. Weizmann Institute of Science (WIS) heliostat field layout.

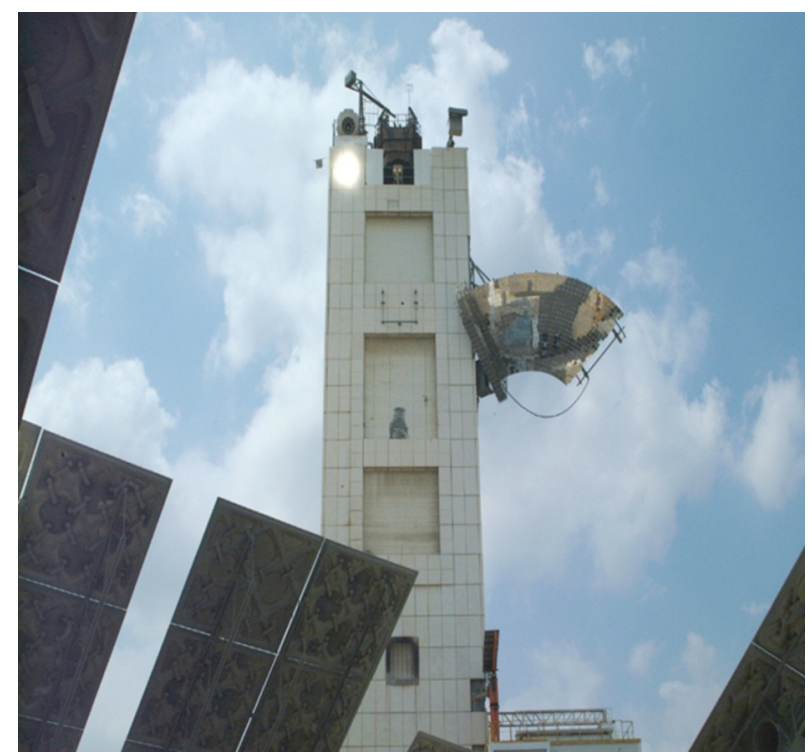

Fig. 3. Weizmann Institute of Science (WIS) solar tower: five experimental stations and an upper mirror for beam-down project.

heliostats to obtain a stationary state in the receiver, the most difficult task in the operation of a solar-powered system that is, essentially, a transitive process. Also, in this framework, the first computer codes with large area of applications were created and validated. Later, these codes became part of a large package called WISDOM [4].
Another pioneer project was connected with the development of the world's first solar-operated Brayton cycle with gas turbine and ceramic tube solar receiver on a $500-\mathrm{kW}$ scale. A first-of-its-kind cavity receiver with $\mathrm{SiC}$ tubes and solar heating of air at about 10 bars and $950{ }^{\circ} \mathrm{C}$ was used. For the first time, electricity with a good total efficiency was produced in our facilities [5].

\section{Storage of solar energy}

In the early nineties, the direction of our research changed. We realized that the technology for production of electricity using solar towers was sufficiently well known and there was no need for new research in that field. We began researching a different approach to converting solar energy, involving capturing sunlight, converting it to high temperature heat, and using this heat to initiate certain chemical reactions. The results indicate several possibilities for such solar-driven reactions, in addition to providing the key to the solution of two of the major hurdles blocking solar energy implementation - its storage and transmission over long distances. Such applications require a chemical system that can absorb large amounts of solar energy to generate suitable products that can be easily stored and transported by conventional means.

The particular chemical systems under investigation by our team are known in the industry as "reforming reactions" $[6,7]$. In these transformations, a hydrocarbon, such as methane, is mixed with steam or carbon dioxide at high temperature (up to $850{ }^{\circ} \mathrm{C}$ ) in the presence of suitable catalysts. Large amounts of heat are absorbed, producing a mixture of hydrogen and carbon monoxide. This combination is known as "synthesis gas". The technology for its storage and transmission is well known.

A particular property of synthesis gas, as far as its application to large scale use of solar energy is concerned, is the fact that its formation can be reversed, returning to the starting materials along with almost all of the energy obtained from the sun. It is thus possible to construct a closed-loop operation in which solar energy is used to producesynthesis gas in a remote desert region. This is then piped to the distant user, where the reaction is reversed, with the energy contained in the system released as heat and the starting materials returned by pipes to the desert solar plant. Such a closed cycle does not consume or emit any chemicals and only serves to transport solar energy captured in one location and deliver it to a distant user. This concept is known as the "chemical heat pipe" and was first suggested and tested in Germany as a possible way of transporting nuclear generated heat from remote reactors to industrial centers. Our group has demonstrated the feasibility of this concept with solar energy, and loops using a few $100 \mathrm{kw}$ have been successfully operated. Our preferential reaction for this storage is the reaction of methane reforming: $\mathrm{CH}_{4}+$ $\mathrm{CO}_{2} \leftrightarrow \mathrm{H}_{2}+2 \mathrm{CO}$ (enthalpy of reaction: endotherm, $\Delta \mathrm{H}=$ $247 \mathrm{~kJ} / \mathrm{mol}$ ); which is always accompanied by the reverse water gas-shift reaction: $\mathrm{CO}_{2}+\mathrm{H}_{2} \leftrightarrow \mathrm{CO}+\mathrm{H}_{2} \mathrm{O}(\Delta \mathrm{H}=-36$ $\mathrm{kJ} / \mathrm{mol}$ ). The maximum operating pressure and temperature of the WIS tubular reformer were 10 to 15 bars and 800 to $850^{\circ} \mathrm{C}$. Both reactions require the presence of a specific catalyst; a ruthenium on stabilized alumina substrate was successfully developed [8]. 
In order to demonstrate the feasibility of this storage, a pilot installation for the methanation (the inverse reaction of the reforming) was built close to the solar tower. Thereby, a chemical heat pipe based on solar energy was put in operation for the first time in the world.

Our team also investigates more traditional processes, such as solar thermal storage using phase-change materials. The thermal storage medium is made of a $\mathrm{Zn}-\mathrm{Sn}$ alloy, which serves as the phase-change material. This development was specifically related to the parabolic trough technology and was tested in a $30 \mathrm{kWh}$ storage capacity and $10 \mathrm{~kW}$ power rate to produce saturated steam at 70 bars. The discharge of the stored heat was achieved by boiling of a commercial organic heat transfer fluid on the phase-change materials and condensation on the external steam generator [9].

\section{Carbothermal reduction of metal oxides; solar thermochemical hydrogen}

During the last decade, special attention has been paid to carbothermal reduction of metal oxides as an efficient candidate for storage and transport of solar energy and also for thermochemical hydrogen production. The metals obtained by carboreduction can be efficiently used in reaction of water splitting in order to obtain hydrogen. In industry, these reactions require a considerable amount of energy and produce a vast amount of carbon dioxide and other pollutants. For these reasons, this process is inconvenient for conventional industry. In contrast, using solar energy, these reactions are environmentally clean. However, nearly all hydrogen used today is produced by means of expensive processes that require combustion of polluting fossil fuels. Moreover, storing and transporting hydrogen is extremely difficult and costly. The new solar technology tackles these problems by creating an easily storable intermediate energy source from metal ore, such as metal oxides.

Schematically, the production of hydrogen is based on two-step water-splitting cycle. In the first step, the metal oxide $\left(\mathrm{Me}_{\mathrm{n}} \mathrm{O}_{\mathrm{m}}\right)$ is dissociated, in an endothermic reaction, in metal $(\mathrm{Me})$ and oxygen $(\mathrm{O}): \mathrm{Me}_{\mathrm{n}} \mathrm{O}_{\mathrm{m}} \rightarrow \mathrm{nMe}+$ $\mathrm{m} / 2 \mathrm{O}_{2}$. This reaction is made in a solar reactor. Because, in most cases, this reaction requires high temperatures, sometimes greater than $1200{ }^{\circ} \mathrm{C}$, the solar receivers must be provided with concentrators at the entrance (see next section). The second step (non-solar) is an exothermic reaction, the hydrolysis of the metal at relatively moderate temperature (about $500^{\circ} \mathrm{C}$ ) to obtain hydrogen: $(\mathrm{nMe}+$ $\mathrm{mH}_{2} \mathrm{O} \rightarrow \mathrm{Me}_{\mathrm{n}} \mathrm{O}_{\mathrm{m}}+\mathrm{mH}_{2}$ ). It is possible to observe that adding both equations: $\mathrm{H}_{2} \mathrm{O} \rightarrow \mathrm{H}_{2}+\frac{1}{2} \mathrm{O}_{2}$. This process has multiple advantages: if its purpose is to dissociate the metal oxide in order to obtain respective metals, the process is complete after the first step; if its purpose is to obtain hydrogen, the hydrogen and the oxygen are obtained in two separate reactions; therefore, here the need for high temperature separation is eliminated [10].

Numerous tests were performed in the WIS solar facilities with different metal oxides and different metal hydrolysis reactions. Zinc oxide was selected as an intermediate promising substance for both reactions. This was confirmed by the results of the European pilot scale SOLZINC project [11], collaboration between scientists from WIS and from Switzerland, France and Sweden.

The SOLZINC reactor was operated with $350 \mathrm{~kW}$ solar input and it produced about $50 \mathrm{~kg}$ per hour $\mathrm{Zn}$ powder from $\mathrm{ZnO}$, at about $1200{ }^{\circ} \mathrm{C}$ in the reaction zone. The resultant zinc can be easily stored, transported, and converted to hydrogen on demand. In addition, the zinc can be used directly, for example, in Zn-air batteries, which serve as efficient converters of chemical- to electrical energy. Thus, this method offers a way of storing solar energy in chemical form and releasing it as required [11].

Another way for production of hydrogen studied in our solar complex was methane decomposition. In solar cracking, methane can be thermally split into hydrogen and carbon black via the following reaction: $\mathrm{CH}_{4} \rightarrow \mathrm{C}$ (solid) $+2 \mathrm{H}_{2}\left(\Delta \mathrm{H}_{298}=75 \mathrm{~kJ} / \mathrm{mol}\right)$. The dissociation starts at about $700 \mathrm{~K}$ and approaches completion at about $2000 \mathrm{~K}$. Promising results were received during the international SOLHYCARB project on solar thermal splitting of methane to hydrogen and solid carbon in the form of nanoparticles [12].

\section{Volumetric receiver}

Most of the solar receivers can be classified as either indirectly irradiated or directly irradiated (volumetric). The common characteristic of the indirectly irradiated receivers is that the heat transfer to the working fluid does not take place upon the surface, which is exposed to incoming solar radiation. Instead, there is an intermediate wall, which is heated by the irradiated sunlight on one side and transfers the heat to a working fluid on the other side. Due to material and design limitations, an increase in the operating temperature poses more severe restrictions on the pressure and the solar flux. For example, at the upper temperature range of indirectly irradiated receivers $(\mathrm{T}$ $>800{ }^{\circ} \mathrm{C}$ ) the flux and pressure cannot exceed $600 \mathrm{~kW} / \mathrm{m}^{2}$ and 10 bars, respectively.

In volumetric receivers, the heat transfer to the working fluid takes place upon the surface, which is heated directly by incoming radiation. Receivers of this diverse group have been developed for both parabolic dish and central receiver systems. In general, the flux, working pressure and temperature achieved by these receivers have not exceeded those of tubular receivers. The only receiver so far to demonstrate a significant advance in operating conditions is the receiver developed by our team and named: Directly Irradiated Annular Pressurized Receiver (DIAPR), tested successfully under incident flux of over $5000 \mathrm{~kW} / \mathrm{m}^{2}$, while delivering air at a temperature and pressure of $1200^{\circ} \mathrm{C}$ and 20 bars, respectively (Fig. 4).

The solar radiation, concentrated by a compound parabolic concentrator (CPC) mounted at the entrance of the receiver, is transmitted into the receiver through a quartz window, which withstood, without damage, a few 100 hours of repeated experiments. The absorber is an array of a few 100 pin-fins (Fig. 4) implanted in a base plate. 


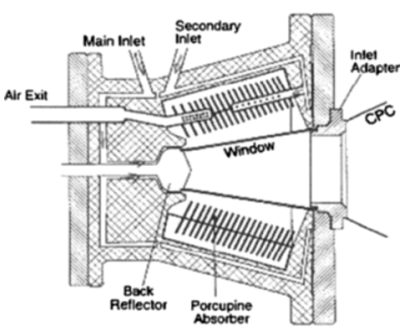

Fig. 4. Directly Irradiated Annular Pressurized Receiver (DIAPR) volumetric receiver [13].

The orientation of the pins is in the general direction of incoming radiation and the mean flow direction is perpendicular to them. Thus, the convective of the heat transfer fluid can be matched better with the irradiation flux distribution over the absorber.

\section{Optical concentrators 6.1 Compound parabolic concentrators}

Each project presented in the previous sections requires a certain operating temperature and a certain amount of solar energy into a receiver. But, at pilot scale (a few $100 \mathrm{~kW}$ ) these two requirements cannot be carried out simultaneously. In order to collect a large amount of energy the receiver aperture must be larger, but at that aperture a large amount of energy will be lost by reradiation. In most cases, a solar concentrator adapted at the receiver aperture is a satisfactory solution. A solar concentrator has a large entrance area capable of collecting the required power from the heliostat field and a small exit area coupled with the receiver aperture, in order to decrease the loss by reradiation. The most suitable concentrator is the CPC [14]. Referring to Figure 5, all rays entering at the extreme collecting angle $\theta_{\max }$ will emerge through point $\mathrm{F}$ of the exit aperture [14]. If we restrict ourselves to rays in the meridian section, the solution is simple, since it is well known that a parabolic shape with its axis parallel to direction $\theta_{\text {max }}$, and its focus at $\mathrm{F}$, will correspond to the geometrical requirement. The complete concentrator must have a symmetrical axis if it is to be a $3 \mathrm{D}$ system, so the reflecting surface is obtained by rotating the parabola about the concentrator axis (not about the axis of the parabola). 3D-CPC is completely defined by the acceptance angle $\theta_{\max }$ and the entrance $d_{1}$ or exit $d_{2}$ apertures (Fig. 5). It is known that: maximum concentration $C_{\max }=1 / \sin ^{2} \theta_{\max }$; length: $L=\left(d_{1}+d_{2}\right) /\left(2 \tan \theta_{\max }\right)$. The dimensions of $d_{1}$ or $d_{2}$ and the acceptance angle $\theta_{\max }$ are defined by a complex connection between the CPC entrance area, the power that must be collected from the heliostat field (at least at design point) and the heliostat field layout. If the heliostat field exists, the requirements for collected power must be adjusted in conformity with the existing heliostat layout, but, when the heliostat field is in the design stage, these requirements must be satisfied by accurate design. Moreover, for big CPCs, the manufacturing of an exact profile entails serious technical difficulties; therefore, most

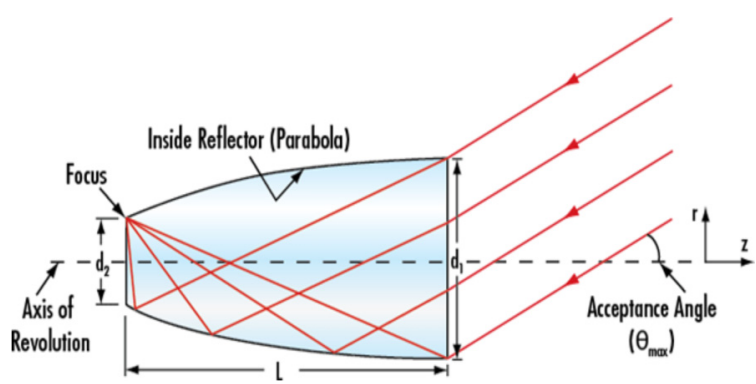

Fig. 5. 3D-compound parabolic concentrator (CPC) scheme.
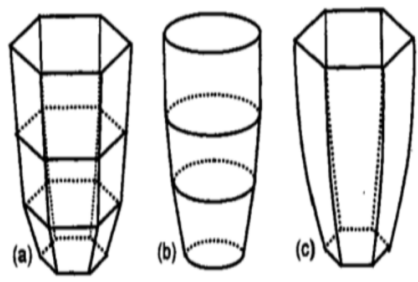

Fig. 6. Compound parabolic concentrator (CPC) approximated by: (a) truncated pyramids; (b) truncated cones; (c) bended planar sheets.

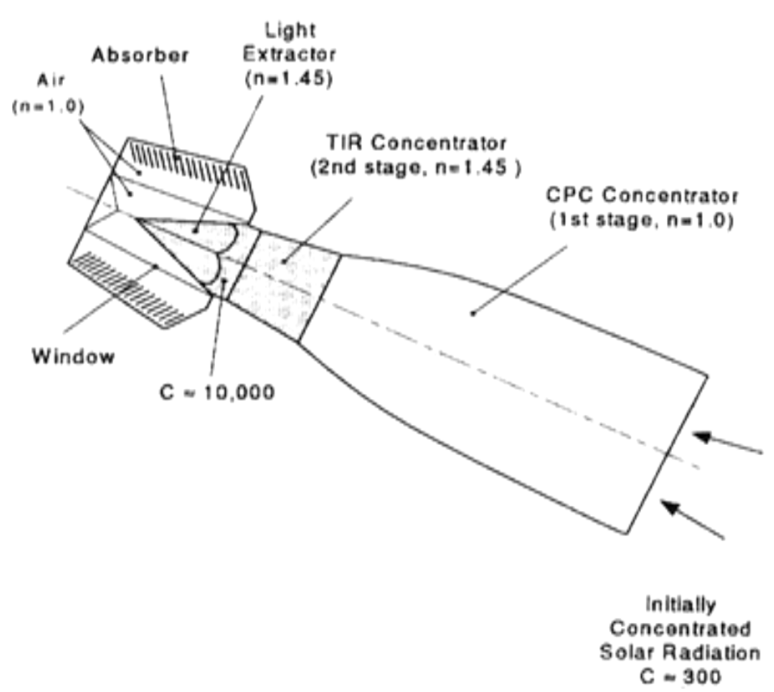

Fig. 7. Two-stage concentrator with light extractor [15].

cases resort to an approximate mathematical profile. Our group has a remarkable experience in approximation of a perfect CPC by a series of truncated pyramids, cones or initially by planar sheets curved so that their edges coincide with the mathematical profile (Fig. 6).

\subsection{Two-stage 3D-CPC}

In order to increase the concentration in a 3D-CPC with interior mirror walls, a next stage dielectric is attached at the end of that CPC. The dielectric CPC is designed so that each ray hitting the contact surface between dielectric and air will be totally internally reflected (TIR on Fig. 7). In order to avoid the TIR at the exit from this dielectric stage, 


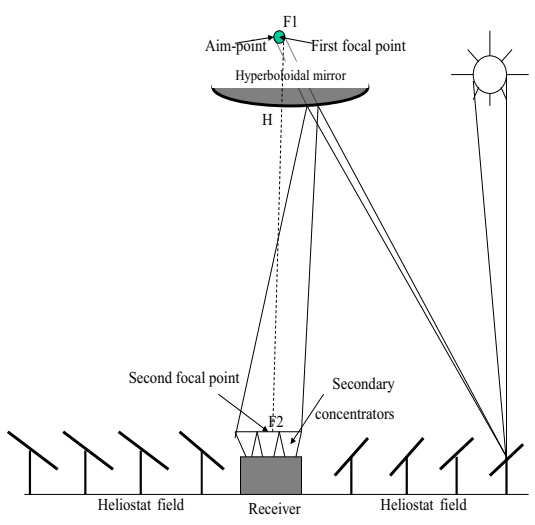

Fig. 8. Beam-down principle.

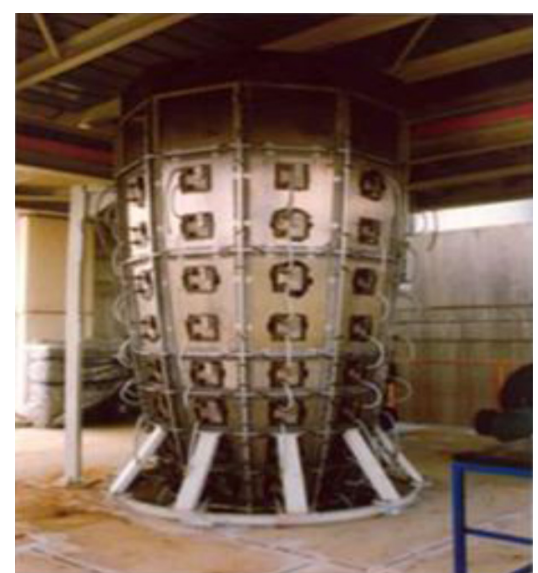

Fig. 9. The biggest concentrator from the world (5-meter height; 2.2-meter entrance diameter).
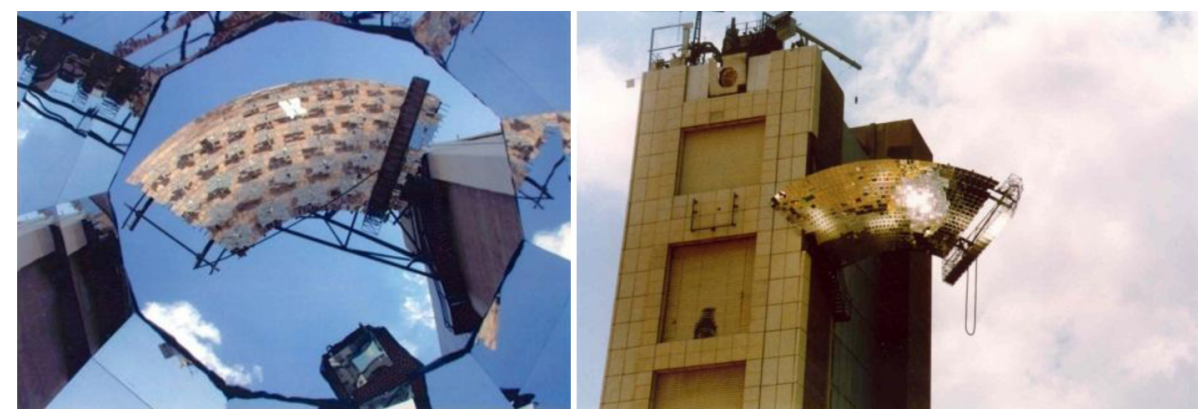

Fig. 10. The upper mirror (left: viewed from the compound parabolic concentrator [CPC] exit).

an extractor was invented [15]. With this tandem of concentrators-extractor, a concentration of 10,000 suns for a power of about $100 \mathrm{~kW}$ has been achieved.

\section{Beam-down concept}

Usually in large solar power plants the receiver and the turbine together with auxiliary equipment are too heavy a load to be supported on the top of a tower structure. An alternative option is to invert the path of the solar rays originating from a heliostat field in such a way that the solar receiver and the above-mentioned equipment can be placed on the ground. In order to carry out this optical path inversion, a supplementary reflector has to be installed. This causes the rays oriented to the aim point of the field to be reflected down to the receiver concentrator (RC) entrance located near the ground [16]. From an optical point of view, only a reflective surface having two foci is capable of this mission, namely, each ray that is oriented to one of its foci (which coincides with the aim point of the heliostat field) will be reflected to the second focus positioned at the entrance plane of the RC. From mathematical point of view, this surface is a quadric, namely, a hyperboloid (with two sheets, of which only the upper one is used) or an ellipsoid. The comparison made shows that the hyperboloidal surface is more promising than the ellipsoidal one [17]. In this mode, we arrived at a new optics named beam-down or tower reflector optics that is today an important challenge for traditional optics. In a series of papers [18-21], we established the most optical aspects of this novelty. In order to prove this idea, construction of a pilot station for $0.7 \mathrm{MW}$ thermal power in WIS solar complex was started at the beginning of 1999. These unique optics include a tower reflector shaped as a section of hyperboloid revolution with one sheet, having a reflective surface area of about $75 \mathrm{~m}^{2}$, and a ground level secondary CPC with 2.2-meter entrance diameter and 5meter height and is capable of enhancing the incoming radiation by a magnification factor of 25 , providing, at the design point, an amount of power about $650 \mathrm{~kW}$ at an average concentration of about 4000. This secondary concentrator, which was built in 2000, remains the biggest of its kind in the world (Figs. 8-10).

Solar tests were carried out successfully throughout 2001 in order to demonstrate the feasibility of a large-scale central solar receiver for future solar power plants.

\section{Conclusions}

The Weizmann Institute's solar research complex is one of the world's most advanced, sophisticated and multiplediscipline facilities for the implementation of concentrated solar energy. Our research was always supported by international and national programs and finance, and results obtained during last 25 years' activity are widely used in the solar community. 


\section{References}

1. D. Barlev, R. Vidu, P. Stroeve, Innovation in concentrated solar power, Sol. Energy Mater. Sol. Cells 95, 2703 (2011)

2. M. Epstein, I. Vishnevetsky, A. Segal, R. Rubin, D. Lieberman, Research and development in the Solar Research Facilities Unit of the Weizmann Institute of Science: past, present and future, Int. J. Environ. Sustain. 9, 97 (2014)

3. M. Epstein, D. Lieberman, M. Rosh, A. Shor, Solar testing of $2 \mathrm{MW}$ water/steam receiver at the Weizmann Institute Solar Tower, Sol. Energy Mater. 24, 265 (1991)

4. A. Segal, WISDOM-Weizmann Institute Solar Dedicated cOmprehensive Mastercode, in Proceedings of the Solar 96 ASES Annual Conference, Asheville, NC 308 (1996)

5. M. Epstein, Solar tests of a ceramic tubes receiver at the Weizmann Institute of Science Solar Research Facilities: Status report, in Proceeding of the 4th International Symposium on Solar Thermal Technology: Research, Development and Applications, Santa Fe, NM (Hemisphere Pub. Co., New York, 1988), 231

6. M. Levy, R. Levitan, H. Rosin, R. Rubin, Solar energy storage via a closed-loop chemical heat pipe, Sol. Energy 50, 179 (1993)

7. A. Segal, M. Epstein, Solar ground reformer, Sol. Energy 75, $479(2003)$

8. A. Berman, K.K. Rakesh, M. Epstein, A new catalyst system for high-temperature solar reforming of methane, Energy Fuels 20, 455 (2006)

9. R. Adinberg, D. Zvegilsky, M. Epstein, Heat transfer efficient thermal energy storage for stem generation, Energy Convers. Manag. 51, 9 (2010)

10. I. Vishnevetsky, A. Berman, M. Epstein, Features of solar thermochemical redox cycles for hydrogen production from water as a function of reactants' main characteristics, Int. J. Hydrog. Energy 36, 2817 (2011)
11. C. Wieckert, U. Frommherz, S. Kraupl, E. Guillot, G. Olalde, M. Epstein, S. Santen, T. Osinga, A. Steinfeld, A 300-kW solar chemical pilot plant for the carbothermal production of zinc, J. Sol. Energy Eng. 129, 190 (2007)

12. M. Kogan, A. Kogan, Production of hydrogen and carbon by solar thermal methane splitting, Int. J. Hydrog. Energy 28, 1187 (2003)

13. J. Karni, A. Kribus, P. Doron, R. Rubin, A. Fiterman, D. Sagie, The DIAPR: a high-pressure, high temperature solar receiver, J. Sol. Energy Eng. 119, 74 (1997)

14. J.J. O'Gallagher, Nonimaging optics in solar energy (Morgan and Claypool Publishers, 2008)

15. H. Ries, A. Segal, J. Karni, Extracting concentrated guided light, Appl. Opt. 36, 2869 (1997)

16. A. Segal, M. Epstein, Modeling of solar receiver for cracking of liquid petroleum gas, J. Sol. Energy Eng. 119, 48 (1997) (world priority for beam-down concept)

17. A. Segal, M. Epstein, Comparative performances of tower-top and tower-reflector central solar receivers, Sol. Energy 65, 206 (1999)

18. A. Segal, M. Epstein, "Potential efficiencies of a solar-operated gas turbine and combined cycle, using the reflective tower optics", in Proceedings of ISES Solar World Congress, Jerusalem, Israel (1999)

19. A. Segal, M. Epstein, The optics of the solar tower reflector, Sol. Energy 69, 229 (2000)

20. A. Segal, M. Epstein, Practical considerations in designing large scale beam-down optical systems, J. Sol. Energy Eng. 130, 011009 (2008)

21. A. Segal, M. Epstein, Truncation of the secondary concentrator (CPC) as means to cost-effective beam-down system, J. Sol. Energy Eng. 132, 031004 (2008)

Cite this article as: Akiba Segal, Solar energy at high temperatures; researches at the Weizmann Institute of Science, Israel; 25 years of success, Renew. Energy Environ. Sustain. 1, 1 (2016) 